In Cres. Vol. $3 N^{\circ}$ 2: pp. 277-287, 2012

\title{
CLIMA ORGANIZACIONAL Y DISFUNCIONES EMOCIONALES EN LOS PROFESIONALES DE ENFERMERÍA DEL HOSPITAL III CHIMBOTE - 2009*
}

\author{
ORGANIZATIONAL CLIMATE AND EMOTIONAL DYSFUNCTIONS \\ IN NURSES AT ESSALUD III HOSPITAL - CHIMBOTE-2009
}

Nhuria Cecilia Rocano Susanibar ${ }^{1}$, Abner Fonseca Livias ${ }^{2}$

\begin{abstract}
RESUMEN
La presente investigación se efectuó en el año 2009, en el Hospital III Es Salud Chimbote, Perú, con el objetivo de establecer la relación entre el clima organizacional y las disfunciones emocionales que perciben los profesionales de Enfermería que laboran en dicha institución. Es un estudio correlacional, prospectivo, de corte transversal, conformada por una población muestral de 68 enfermeros, quienes respondieron a las entrevistas, escalas, inventarios, el test del clima organizacional y disfunciones emocionales. El análisis estadístico empleado fue la $\mathrm{X}^{2}$ y la r de Pearson apoyándonos en el SPSS V17. La investigación determinó que el clima social en el trabajo y las disfunciones emocionales expresan cualitativamente correlación alta, $[(\mathrm{x} 2=26,17 \mathrm{GL}=2 ; \mathrm{p}=0,000)]$; cuantitativamente revela correspondencia moderada negativa y descendente $[(\mathrm{r}=-0,643, \mathrm{p}=0,000)]$, correlación inversamente proporcional, lo que indica que, mientras mejor se perciba el clima organizacional, menores disfunciones emocionales se presentarán y viceversa. Un importante número de profesionales de Enfermería perciben un clima organizacional positivo. Existe una relación bastante significativa entre el clima organizacional y las disfunciones emocionales en los profesionales de Enfermería.
\end{abstract}

Palabras Clave: Clima organizacional, Disfunciones emocionales, Profesionales de Enfermería, Salud ocupacional.

* Recibido: 04 de mayo del 2012; aprobado: 25 de octubre del 2012.

1 Magister en Administración y Gerencia de los Servicios de Salud. Docente de la Escuela Profesional de Enfermería de la Universidad Católica Los Ángeles de Chimbote. Enfermera Asistencial en La Unidad Crítica de Neonatología del Hospital III EsSalud Chimbote. Especialista en Cuidados de la Mujer y Recién Nacido.

2 Doctor en Enfermería. Docente Principal de la Facultad de Enfermería de la Universidad Nacional Hermilio Valdizán. 


\section{ABSTRACT}

This retrospective cross sectional descriptive study was performed at Hospital III Es Salud Chimbote, Perú aimed to establish the relationship between emotional dysfunction and organizational climate. The sample population consisted of 68 nurses, the ones who participated in interviews, scales, inventaries, organizacional climate and emotional dysfunction test. The SPSS version 17.0 was used for dataprocessing. Results were statistically analyzed by the X2 and Pearson's correlation coefficient.

The study results showed that a significant number of nurses perceive a positive organizational climate and concluded that there is a significant corelation between emotional dysfunction and organizational climate $[(\mathrm{x} 2=26,17 \mathrm{GL}=2 ; \mathrm{p}=0,000)][(\mathrm{r}=-0,643$, $\mathrm{p}=0,000)$ ], implying that an inverse relationship was established with these two variables. So, the better the organizational climate is perceived the less emotional dysfunction cases and viceversa.

KEY WORDS: Organizational climate, Emotional dysfunction, Nurses, Occupational health.

\section{INTRODUCCIÓN}

En la actualidad, una manera de contribuir al desarrollo de las organizaciones, es mejorar la calidad de vida de los empleados en el ámbito de trabajo, dado que, en tanto el trabajador perciba una sensación de bienestar físico y psicosocial, ello se verá reflejado en una actitud favorable o desfavorable hacia su trabajo. ${ }^{1,2}$ Tal es el caso particular de los profesionales de Enfermería, que por la naturaleza de su trabajo, se demandan competencias elevadas en el manejo de situaciones críticas y un desempeño eficiente y efectivo al tratar con vidas humanas; por lo que se encuentra catalogada como una profesión expuesta a múltiples riesgos y altamente estresante ${ }^{3,4,5}$, resultando uno de los eventos perjudiciales para su salud las disfunciones emocionales los que generan un deterioro progresivo de sus funciones orgánicas. ${ }^{6,7}$ En este sentido, resulta relevante orientar acciones de salud ocupacional en aras de dotar a dichos trabajadores un clima organizacional positivo. ${ }^{8}$

El objeto de estudio de la presente investigación es la salud laboral de los profesionales de Enfermería, ya que es uno de los aspectos para mejorar la funcionalidad eficiente de las organizaciones de salud; por tanto, el desarrollo de climas organizacionales favorables es fundamental para este grupo ocupacional, ya que está demostrado que el trabajo en un clima adecuado favorece una actitud de mayor compromiso con la excelencia y la calidad final en los productos o servicios que se ofrecen, con el consiguiente impacto en la actividad productiva y en el bienestar emocional de sus trabajadores. ${ }^{8,9,10}$ 
Algunos estudios de campo realizados por Ribera, Cartagena, Reig, Romá, Sans, Caruana ${ }^{11}$, sobre estrés percibido en los profesionales de Enfermería encuestados fue de $75.7 \%$ en mayor o menor grado. Un importante grupo $(19,8 \%)$ expresa estar padeciendo un estrés máximo, mientras que el resto $(65,9 \%)$ percibe un estrés débil o moderado y los días de baja por dicha enfermedad entre 1 y 8 días fue de $18,7 \%$. Sin embargo, cuando no se realizan intervenciones oportunas para mejorar el afrontamiento al estrés, se presenta un agotamiento que constituye la fase final de la excesiva exposición a situaciones críticas denominada Sd. Burnout. Tal es el caso de los resultados presentados por otros estudios, donde señalan que se encontró que los niveles y prevalencia de Burnout fue de $62 \%$ para cansancio emocional elevado, un $66 \%$ y $52 \%$ un nivel importante de despersonalización y un $66 \%$, una baja realización perso$\mathrm{nal}^{12}$. No hemos de olvidar que muchas de estas disfunciones emocionales son originadas en el ámbito laboral, por lo que se intenta demostrar que en tanto el trabajador perciba un adecuado o saludable clima laboral, existirán menores disfunciones emocionales. Lo sustentan otros estudios, donde mencionan que los trabajadores perciben bienestar cuando existe equidad, condiciones propias del trabajo y la buena relación con los colegas. Además, la cultura, las actitudes, los comportamientos y valores compartidos por una organización que son de suma importancia para satisfacer las expectativas del trabajador. ${ }^{13}$

\section{PROBLEMA}

¿Cuál es la relación entre el clima organizacional y las disfunciones emocionales que perciben los profesionales de enfermería del Hospital EsSalud Nivel III- Chimbote 2009?

\section{OBJETIVOS}

Establecer la relación entre el clima organizacional con las disfunciones emocionales que perciben los profesionales de enfermería en estudio.

\section{METODOLOGIA}

El estudio fue explicativo, correlacional de corte transversal. La población estuvo conformada por la totalidad de profesionales de Enfermería que laboran en el Hospital III EsSalud - Chimbote, los que son un total de 68 profesionales encuestados durante los meses de marzo a julio del 2009.

Los instrumentos aplicados fueron: Guía de entrevista de las características socio-demográficas y laborales de los profesionales de Enfermería; Escala 
de apreciación de estrés socio-laboral de $\operatorname{Karasek}^{14}$; Inventario de depresión de Beck, creado por Beck, Mendelson, Mock ${ }^{15}$ y traducido al español por Vázquez, Sanz ${ }^{16,17}$. Es una escala tipo Likert, creado por Spielberg, Gorsuch, Lushene ${ }^{17}$. Para medir el grado de disfunciones emocionales, fue necesario tomar los valores netos de las tres escalas anteriormente mencionadas (estrés, depresión y ansiedad); luego se procedió a comprobar si existía relación monótona (Rho de Spearman) entre las tres dimensiones de lo que llamaremos, en general, variable disfunciones emocionales. Posteriormente elevamos dichos valores puros a un perfil correspondiente a cada una, tomando como máximo valor la unidad; en otras palabras, se igualaron los valores correspondientes de las tres escalas. Finalmente, la escala de clima social en el trabajo, Work Environment Scale (WES) de Moos R.H., fue adaptada al castellano en España por Fernández Ballesteros ${ }^{18}$. Las encuestas fueron aplicadas personalmente por la autora en el lugar de trabajo de cada una de las enfermeras. Se garantizó el anonimato y confidencialidad a las enfermeras participantes.

\section{RESULTADOS}

Sobre los resultados obtenidos sobre las características laborales de los profesionales de enfermería en estudio, tenemos que el mayor porcentaje de ellos [73,5\% (50)] son contratados; el 95,6\% (65) recibe su salario por planillas; el nivel de estudios más destacado es de licenciatura [45,6\% (31)] y especialista [42,6\% (29)]; la función asistencial fue la predominante [92,6\% (63)] en casi toda la población de estudio. Asimismo, el haber trabajado por más de 10 años [47,1\% (32)]; el atender de 11 - 20 pacientes por turno [48,5\% (33)]; y el turno rotatorio $[89,7 \%$ (61)] fueron las característica de mayor preponderancia, tal como se muestra en la Tabla 02.

En cuanto a la percepción global del clima social por los profesionales de enfermería en estudio, la mayoría [64,7\% (44)] de dichos profesionales percibieron que sus actividades laborales las desarrollan en un clima social positivo. Destaca un respetable porcentaje [35,3\% (24)] de enfermero(as) que consideran el clima social como negativo.

El clima social en el trabajo y las disfunciones emocionales en los profesionales de Enfermería en estudio, tenemos que: porcentajes considerables de los profesionales que se encontraron en un clima social negativo, ostentaron niveles de disfunciones emocionales leve [2,9\% (2)], moderado [25,0\% (17)] y grave $[7,4 \%(5)]$. 
Tabla 01

CARACTERÍSTICAS LABORALES DE LOS PROFESIONALES

DE ENFERMERÍA EN ESTUDIO. HOSPITAL III

ESSALUD CHIMBOTE - 2009

\begin{tabular}{|c|c|c|}
\hline \multirow[b]{2}{*}{ Características laborales } & \multicolumn{2}{|c|}{$(n=68)$} \\
\hline & $\mathrm{N}^{0}$ & $\%$ \\
\hline \multicolumn{3}{|l|}{ Condición laboral } \\
\hline Contratado/a & 50 & 73,5 \\
\hline Nombrado/a & 18 & 26,5 \\
\hline \multicolumn{3}{|l|}{ Tipo de salario } \\
\hline SNP & 3 & 4,4 \\
\hline Planillas & 65 & 95,6 \\
\hline \multicolumn{3}{|l|}{ Nivel de estudios profesionales } \\
\hline Licenciada & 31 & 45,6 \\
\hline Especialista & 29 & 42,6 \\
\hline Magister & 8 & 11,8 \\
\hline \multicolumn{3}{|l|}{ Función actual } \\
\hline Asistencial & 63 & 92,6 \\
\hline Ambas & 5 & 7,4 \\
\hline \multicolumn{3}{|l|}{ Tiempo de servicio } \\
\hline 1-3 años & 17 & 25,0 \\
\hline 7-10 años & 19 & 27,9 \\
\hline 10 años a más & 32 & 47,1 \\
\hline \multicolumn{3}{|l|}{ Pacientes atendidos por turno } \\
\hline$<11$ & 16 & 23,5 \\
\hline 11 a 20 & 33 & 48,5 \\
\hline 21 a 30 & 4 & 5,9 \\
\hline$>30$ & 15 & 22,1 \\
\hline \multicolumn{3}{|l|}{ Turno en que labora } \\
\hline Turno de mañanas & 7 & 10,3 \\
\hline Turno rotatorio & 61 & 89,7 \\
\hline Total & 68 & 100,0 \\
\hline
\end{tabular}

Fuente: Guía de entrevista de las características socio demográficas y laborales. 
Tabla 02

DISFUNCIONES EMOCIONALES EN LOS PROFESIONALES

DE ENFERMERÍA EN ESTUDIO

\begin{tabular}{lrr}
\hline Disfunciones emocionales & \multicolumn{2}{c}{$\mathbf{N}^{\mathbf{( n = 6 8 )}}$} \\
\hline Leve & 32 & 47,1 \\
Moderado & 31 & 45,6 \\
Elevado & 5 & 7,4 \\
\hline Total & 68 & 100,0 \\
\hline
\end{tabular}

Fuente: Escala de apreciación de estrés socio-laboral de Karasek. Inventario de Beck para la depresión. Cuestionario de ansiedad estado-rasgo.

Tabla 03

PERCEPCIÓN GLOBAL DEL TIPO DE CLIMA SOCIAL EN EL TRABAJO EN LOS PROFESIONALES DE ENFERMERÍA EN ESTUDIO

\begin{tabular}{lcc}
\hline Clima social en el trabajo & $\mathbf{N}^{\mathbf{( n}=\mathbf{6 8})}$ & $\%$ \\
\hline Positivo & 44 & 64,7 \\
Negativo & 24 & 35,3 \\
\hline Total & 68 & 100,0 \\
\hline
\end{tabular}

Fuente: Escala de clima social en el trabajo (WES).

Tabla 04

CLIMA SOCIAL EN EL TRABAJO Y DISFUNCIONES EMOCIONALES EN LOS PROFESIONALES DE ENFERMERÍA EN ESTUDIO

\begin{tabular}{lrrrrrr}
\hline \multirow{2}{*}{ Clima social en el trabajo } & \multicolumn{9}{c}{ Disfunciones emocionales } \\
\cline { 2 - 8 } & \multicolumn{2}{c}{ Leve } & \multicolumn{2}{c}{ Moderado } & \multicolumn{2}{c}{ Grave } \\
\cline { 2 - 8 } & $\mathbf{N}^{\mathbf{0}}$ & $\mathbf{\%}$ & $\mathbf{N}^{\mathbf{0}}$ & $\mathbf{\%}$ & $\mathbf{N}^{\mathbf{0}}$ & $\boldsymbol{\%}$ \\
\hline Positivo & 30 & 44,1 & 14 & 20,6 & 0 & 0,0 \\
Negativo & 2 & 2,9 & 17 & 25,0 & 5 & 7,4 \\
\hline Total & 32 & 47,1 & 31 & 45,6 & 5 & 7,4 \\
\hline
\end{tabular}

Fuente: Escala de apreciación de estrés socio-laboral de Karasek. Inventario de Beck para la depresión. Cuestionario de ansiedad estado-rasgo. Escala de clima social en el trabajo, work environment scale (WES). 
En la primera aproximación de la relación cualitativa de las variables generales: clima social en el trabajo y disfunciones emocionales en los profesionales de enfermería estudiados, observamos que existe correlación alta, siendo esta significativa a cualquier nivel $\left[\left(\mathrm{x}^{2}=26,17 \mathrm{GL}=2 ; \mathrm{p}=0,000\right)\right]$; lo que comprueba la correlación y dependencia entre las dos variables.

Los resultados de la investigación muestran que poco más de la mitad de los profesionales de enfermería en estudio [51,1\% (35)] manifiesta estrés de nivel moderado durante sus diversas actividades laborales y un 10,3\% (7) de enfermeros(as) muestra estrés de nivel elevado.

En cuanto al análisis descriptivo de las disfunciones emocionales de manera general en los profesionales de Enfermería en estudio, tenemos que la mayoría de dichos profesionales presentaron disfunciones emocionales leves $[47,1 \%$ (32)] y moderadas [45,6\% (31)]. Aunque un mínimo porcentaje [7,4\% (5)] de enfermero(as) también presentaron disfunciones de nivel elevado.

La presencia de disfunciones emocionales de origen laboral es materia de interés para los responsables de la gestión de recursos humanos, pues ésta tiene repercusiones importantes sobre la organización (como en la: rotación, disminución de la productividad, disminución de la calidad, en el ausentismo, entre otros) y la sociedad en general. Gil ${ }^{19}$ ha observado que el mayor patrón de ocurrencia del agotamiento emocional por el trabajo se da en los profesionales de servicios, quienes prestan una función asistencial o social y que sus consecuencias se ve reflejado en la calidad de la atención que se brinda al usuario o al cliente. En efecto, el profesional de Enfermería de los servicios asistenciales representa uno de los campos laborales con mayor riesgo, no solo a los factores físicos, sino también psicológicos. ${ }^{20}$ De manera general, se confirma que existe relación entre las variables: clima social en el trabajo y disfunciones emocionales en los profesionales de Enfermería estudiados, que desde un punto de vista cualitativo la correlación resultó ser alta y significativa $\left[\left(\mathrm{x}^{2}=26,17 \mathrm{GL}=2\right.\right.$; $\mathrm{p}=0,000)$ ], lo que demuestra la correlación entre dichas variables, aunque esto no es determinante, por cuanto en la relación cuantitativa se observa una correspondencia moderadamente negativa y descendente $[(\mathrm{r}=-0,643$, $\mathrm{p}=0,000)$ ], siendo inversa, lo que traduce que, efectivamente, cuando está presente un clima social positivo en el trabajo, las disfunciones emocionales también disminuyen en sus niveles leve y moderado en el trabajador; sin embargo, al ser moderada la relación, indica que no en todos los casos aparece dicha condición, hecho que se fundamenta en la teoría del clima organizacional o de los sistemas de organización propuesta por Likert ${ }^{21}$ (citado por Brunet) y en la teo- 
ría del comportamiento humano de $\mathrm{Mc} \mathrm{Gregor}^{22}$, quienes establecen que el comportamiento asumido por los subordinados dependen directamente del comportamiento administrativo y de las condiciones organizacionales (acciones administrativas de la organización) que los mismos perciben y, en parte, por sus informaciones, percepciones; esperanzas, capacidades y valores. Por lo tanto, se afirma que es la percepción la que determina el tipo de comportamiento (la emoción, personalidad, autoestima, inteligencia, carácter, motivación, familia, aprendizaje, cultura, entre otros) que un individuo va a adoptar.

Por su parte, $\operatorname{García}^{23}$ ha señalado que el clima social en el trabajo refleja la interacción entre las características personales de cada individuo y de la organización. Esta interacción crea en cada persona una valoración o construcción de aceptación, rechazo, o de tolerancia que influye necesariamente en sus actitudes y comportamientos, y que con el tiempo puede traducirse en logros, tanto en el ámbito personal, como en el social, económico y laboral o en frustraciones (expresadas en las disfunciones emocionales).

Reforzando estas teorías, Litwin, y Stringer ${ }^{24}$ mencionan que el clima organizacional es un filtro por el cual pasan los fenómenos objetivos (estructura, liderazgo, toma de decisiones); por lo tanto, evaluando el clima organizacional se mide la forma como es percibida la organización.

Relacionando estas teorías con los hallazgos del presente estudio, podemos asumir que las características organizacionales del hospital en estudio, influyen en las respuestas emocionales del personal de salud; que de acuerdo a sus percepciones, una mayor proporción de trabajadores considera que existe un clima laboral positivo; sin embargo, otro grupo importante de trabajadores está percibiendo como negativo, lo que automáticamente está contribuyendo a que el trabajador adopte emociones en cierto modo, con tendencias de normalidad, sin dejar de lado la presencia de ciertas desviaciones en las dimensiones de las disfunciones emocionales; que según Concalves ${ }^{25}$, va a repercutir en las motivaciones, las que generan una gran variedad de consecuencias, tales como la productividad, satisfacción, rotación, adaptación ${ }^{26}$, etc.

Coincidiendo con nuestros resultados, Alvarez $^{27}$, en el estudio sobre la cultura y clima organizacional como factores relevante en la eficacia del Instituto de Oftalmología, determinó que el clima organizacional positivo favorece la existencia elevada(o) de: motivación, productividad, compromiso y lealtad de los trabajadores.

Diferente a nuestros resultados tenemos a los de Quintero, Africano, Faría $^{28}$, quienes en su estudio sobre el clima organizacional y desempeño labo- 
ral del personal de vigilancia de la Costa Oriental del Lago (VADECOL), observaron que el clima organizacional en dicha empresa no es beneficioso, ni satisfactorio para un buen desempeño laboral.

Los modelos teóricos de Golembiewski, Munzenrider, $\operatorname{Carter}^{29}$ y Cox, Kuk, Leiter ${ }^{30}$, también consideran que el agotamiento emocional por el trabajo tiene como antecedentes las disfunciones del rol, la falta de salud organizacional, la estructura, la cultura y el clima organizacional. Estos investigadores enfatizan la importancia de los estresores del contexto de la organización.

\section{CONCLUSIONES}

1. Más de la mitad de los profesionales de Enfermería en estudio $[51,1 \%$ (35)] manifiesta estrés de nivel moderado durante sus diversas actividades laborales y un 10,3\% (7) de enfermeros(as) muestra estrés de nivel elevado.

2. La mayoría de profesionales de Enfermería en estudio percibieron que sus actividades laborales las desarrollan en un clima social positivo. Destaca un respetable porcentaje de enfermero(as) que consideran el clima social como negativo.

3. Sobre el clima social en el trabajo y las disfunciones emocionales en los profesionales de Enfermería en estudio, porcentajes considerables de los profesionales que se encontraron en un clima social negativo, ostentaron niveles de disfunciones emocionales leves, moderadas y graves.

4. Las características organizacionales del hospital en estudio influyen en las respuestas emocionales del personal de salud.

\section{REFERENCIAS BIBLIOGRAFICAS}

1 Ponce J, Reyes H, Ponce G. Satisfacción laboral y calidad de atención de enfermería en una unidad médica de alta especialidad. Rev Enfer IMSS; 2006; 14 (2): 65-73.

2 PeIRÓ JM. Tratado de Psicología del trabajo. La actividad laboral en su contexto. Madrid. Ed. Síntesis. 1996.

3 Bowman G, STERn M. Ajuste en la tensión ocupacional: la relación del control percibido a la eficacia de estrategias que hacen frente. Diario de aconsejar la psicología ;1995; 42(3): 294-303.

4 Kagan N, Watson M. Reducción de tensión en el lugar de trabajo: el efectiveness de los programas del psychoeducational. Diario de aconsejar la psicología; 1997; 42(1): 71-78.

5 Meza Benavidez M. Estrés laboral en profesionales de Enfermería: Estudio sobre evaluación cognoscitiva y afrontamiento. Rev. Enf. Binasss. Vol. 25. №1-17. [Consulta: 8 
de marzo de 2008]. Disponible en: http://www.binasss.sa.cr/revistas/enfermeria/ v25n1/7.pdf.

6 Rocano N. Sondeo Rápido de manifestaciones disfuncionales en de enfermería en estudio. Hospital servicio de medicina - Febrero, 2009.

7 CAmpos C, MeZa M. Salud mental y trabajo. Estrés ocupacional en las enfermeras. [Tesis de licenciatura], Universidad de Costa Rica, San José; 1990.

8 Rosas S. Síndrome de desgaste profesional (Burnout) en el personal de enfermería del Hospital General de Pachuca. 2006. Revista Científica Electrónica de Psicología ICSAUAEH- No 02. [Sitio en internet]. [Consulta: 18 de febrero de 2010]. Disponible en: http://dgsa.uaeh.edu.mx/revista/psicologia/IMG/pdf/No_2-5.pdf.

9 Cox T, Kuk G, Leiter M. Burnout, Health, Work Stress and Organizational Healthiness. En W. B. Schaufeli, C. Maslach y T. Marek. Professional Burnout: recent Developments in Theory and research. London: Taylor \& Francis; 1993.

10 MintZberg H. The structuring of organizations. Englewood Cliffs, N.J: Prentice Hall; 1979.

11 Ribera D, Cartagena de la Peña E, Reig Ferrer A, Romà Ferri MT. Estrés y salud percibidos por los profesionales de Enfermería. 2005. p. 32.

12 Martínez García M, Centeno Cortés CA, Sanz Rubiales, Del Valle ML. Estudio sobre el Síndrome de Burnout en Profesionales de Enfermería de Cuidados Paliativos del País Vasco, 2006.

13 Amoros E. Comportamiento Organizacional en busca del desarrollo de ventajas competitivas. Universidad Católica Santo Toribio de Mogrovejo. Escuela de Economía. Lambayeque; 2007.

14 KARASEK R. Job demands, job decision latitude and mental strain: implications for job redesign. Administrative Science Quarterly; 1979.p. 285-307.

15 Beck A, Freeman A. Terapia cognitiva de los trastornos de personalidad. Paidós. 1995.

16 VÁzQuez C, SANZ J. Fiabilidad y valores normativos de la versión española del inventario de depresión de Beck. Clínica y Salud; 1997.p. 403-422.

17 SpIelberg CD, Gorsuch RL, LuShene RE. STAI. Cuestionario de Ansiedad EstadoRasgo. Madrid: TEA; 2003.

18 FERNÁNDEZ BALLESTEROS R. Tecnología en psicología ambiental; 1987.p. 46-80.

19 GIL MONTE PR. El síndrome de quemarse por el trabajo (síndrome de burnout) en profesionales de enfermería. Revista Eletrônica InterAção Psy - 2003; 1 (1) 19-33.

20 PuRIFICACIÓN H. Declaración Curso regular de enfermería de empresa. [en línea] [fecha de acceso 25 de mayo del 2009]; URL disponible en: http://www.enfermeriasalamanca.com/ TRABAJOS_SALUD_LABORAL/SEGURIDAD/Fatiga_mental.PDF.

21 LiKeRT' A. En Brunet L. El Clima de Trabajo en las Organizaciones: Definiciones, diagnóstico y consecuencias. México:Trillas; 1999.

22 MC GREGOR. Teoría del comportamiento humano de El comportamiento humano [internet]. México. [Citado 01 de octubre 2008]. Disponible en: http://www.mitecnologico.com/ Main/ElComportamientoHumano.

23 García Villamizar GE. Clima social en el trabajo [internet]. México. [Citado 01 de octubre 2008]. Disponible en: http://www.upbbga.edu.co/nuestraupb/medios/ brujula37.pdf. 
24 Litwin G, Stringer R. Motivation and Organizational Climate. Boston: Division of Research Graduate school of Business Administration Harvard University; 1968.

25 Concalves Alexis. Dimensiones del clima organizacional. [internet]. Ginebra. [Citado 01 de octubre 2008]. Disponible en: http://www.educadormarista.com/proyectoaprender/ clima-organizacional.htm.

26 Flores Jaime J. Aplicación de los estímulos organizacionales para el mejoramiento del clima organizacional el caso del laboratorio farmacéutico Corporación Infarmasa SA. Lima. 2007. Tesis de postgrado (Tesis para optar el titulo de magister en administración y Gestión Empresarial). Universidad Nacional Mayor de San Marcos. Facultad de ciencias administrativas. Unidad de postgrado.

27 Alvarez Valverde, Shirley Yissela. Op.cit., p. 4-5.

28 Quintero NiRia, Africano Nelly, FARÍA Elsis. Clima Organizacional y Desempeño Laboral del Personal Empresa Vigilantes Asociados Costa Oriental del Lago. En Revista NEGOTIUM / Ciencias Gerenciales.( Abr). 2008; 3 (9): pp 33-51.

29 GolembiewSKi RT, MUNZENRIDER R, CARTER D. Phases of progressive burnout and their work site covariants: Critical issues in OD research and praxis. Journal of Applied Behavioral Science; 1983.p. 461-481.

30 Cox T, KuK G, Leiter M. En: Burnout, health, work stress, and organizational healthiness. En W. B. Schaufeli, C. Maslach y T. Marek (Eds.), Professional burnout: Recent developments in theory and research. London: Taylor \& Francis; 1993.p. 177-193. 